\title{
APPLICABILITY OF EFFECTIVE REGION TECHNIQUE IN ULTRASONIC IMAGING BY AWS-SAFT
}

\author{
Worawit PADUNGSRIBORWORN ${ }^{1}$, Akira FURUKAWA ${ }^{2}$ and Sohichi HIROSE ${ }^{3}$ \\ ${ }^{1}$ Student member of JSCE, Graduate Student, Dept. of Civil Eng., Tokyo Institute of Technology \\ (2-12-1-W8-301, Ookayama, Meguro-ku, Tokyo 152-8550, Japan) \\ E-mail: yamatox20a@yahoo.co.jp \\ ${ }^{2}$ Member of JSCE, Assistant Professor, Dept. of Mechanical and Environmental Informatics, \\ Tokyo Institute of Technology (2-12-1-W8-301, Ookayama, Meguro-ku, Tokyo 152-8550, Japan) \\ E-mail: furukawa.a.aa@m.titech.ac.jp \\ ${ }^{3}$ Member of JSCE, Professor, Dept. of Mechanical and Environmental Informatics, Tokyo Institute of Technology \\ (2-12-1-W8-302, Ookayama, Meguro-ku, Tokyo 152-8550, Japan) \\ E-mail: hirose.s.aa@m.titech.ac.jp
}

\begin{abstract}
In ultrasonic testing, SAFT (Synthetic Aperture Focusing Technique) is a signal processing technique used to reconstruct flaw images by the superposition of A-scan waveforms. However, an additional signal processing technique may also be integrated into SAFT algorithms to get clearer ultrasound images. Wave theory is one of the most significant keys in SAFT improvement because it can lead to a better understanding of the wave phenomenon. In this paper, approximate wave solution (AWS) based on a stationary phase method is used to calculate beam radiations inside a target of inspection. Multiplying A-scan waveforms by the ultrasonic beam radiations computed from the AWS, wave diffraction characteristics that are spatial functions between a transducer and a target are incorporated into the SAFT. Moreover, the AWS can be used to predict the Effective Region (ER) where high imaging capability can be obtained. The performance of the improved SAFT, namely AWS-SAFT, and the ER technique are experimentally tested in ultrasonic imaging for a side-drilled hole (SDH) in a steel plate. As a visualizing aid, Structural Similarity (SSIM) index is introduced and Structural Similarity Index Map (SSIM-MAP) is proposed instead of a SAFT image. It is shown that SSIM-MAP can improve a flaw image, especially in that the image is more focused on the location of a flaw, and artifacts due to a longitudinal wave mode are eliminated in a transverse wave mode SAFT image. The ER, where a high contrast flaw image can be definitely obtained, is defined quantitatively in advance of SAFT imaging. The preliminary study for the use of the proposed technique in a real problem is demonstrated using ultrasound imaging of a bottom defect of a steel plate to demonstrate the application of AWS-SAFT and ER.
\end{abstract}

Key Words: SAFT, phased-array transducers, ultrasonic beam radiation, angle beam testing

\section{INTRODUCTION}

Many crucial infrastructures such as bridges and dams have been constructed since the middle of the 20 th century. In other words, they have been in service for more than 50 years. Properly designed and built structures are supposed to last through their design life specified in many design codes. ${ }^{1), 2)}$ However, without appropriate maintenance, they may experience sudden collapses due to structural deficiencies such as fatigue cracks and corrosions. ${ }^{3)}$ Structural health monitoring ${ }^{4)}$ then becomes important in society because a structural failure can cause catastrophes to lives and assets. Such importance has been drawing mass attention into researches of nondestructive techniques to precisely identify quantita- tive information such as sizes, shapes, and locations of flaws in materials and structures in a harmless manner. ${ }^{5)}$ Among various techniques, ultrasound imaging, which is also used in the medical field, ${ }^{6}$ ) plays an important role in detecting flaws in materials and structures for these reasons: (1) it is safe to humans and the environment, and (2) the devices are portable. To obtain an ultrasound image, a signal processing technique is required to convert the waveform signals acquired from an Ultrasonic Testing (UT) to an ultrasound image. One of the most efficient signal processing techniques is Synthetic Aperture Focusing Technique (SAFT).

SAFT has changed the way of interpretating UT results since its development in 1974 by the US Nuclear Regulatory Commission at the University of 
Michigan. ${ }^{7)}$ SAFT uses the same concept as Synthesis Aperture Radar (SAR), which is an imaging technique utilizing microwave. The principle of this technique was written in detail by Seydel ${ }^{8)}$ and a review of SAFT development was reported by Doctor et al. ${ }^{9)}$ Transverse and longitudinal resolutions of SAFT were studied by Thomson. ${ }^{10)}$ Experiences with SAFT in field measurement were introduced by Schmitz et al. ${ }^{11)}$ The strong points of SAFT lay on its simple and flexible algorithm. Improvement of signal-tonoise ratio (SNR) can be obtained by superposition of signals from multiple transmitter-receiver probe positions, so that flaw echoes buried inside noises become visible.

The invention of phased-array transducers has made the use of SAFT easier since ultrasonic signals at multiple positions can be obtained within a short time. Preliminary use of an array system for the inspection of weldments was done by Lamarre et al. ${ }^{12}$ ) Drinkwater and Wilcox reviewed a meantime research progress in the use of ultrasonic arrays for NDE, offering a step change over traditional monolithic transducers. ${ }^{13)}$ Using phased-array technology, vast amounts of wave data are easily obtained. However, signal processing of such large amounts of data is not a critical task because of rapid computing technology in these days. Recently, a 3D imaging in real time has been developed and performed intensely in medical ultrasound. ${ }^{14)-16)}$

The algorithm of a conventional SAFT is quite simple. However, some improvements for the conventional SAFT algorithm had been investigated by engineers and researchers for decades in both time and frequency domains. In frequency domain, SAFT is treated as a special solution of an inverse scattering problem. ${ }^{17)-19)}$ Another example of SAFT improvement is allowing the use of multiple reflections. ${ }^{20), 21)}$ The most recent state-of-the-art review on SAFT is written by Spies et al. ${ }^{22)}$ Such accomplishments in the improvement of SAFT show that wave theory is a key point in getting more precise flaw images since it can predict the characteristics of utilized ultrasonic waves resulting in better approximation of real phenomena.

This paper attempts to implement ultrasonic beam radiations, which can be explicitly computed using Approximate Wave Solution (AWS) derived by Schmerr, ${ }^{23)}$ into a SAFT algorithm. A preliminary study on this idea was done by Hirose et al. in fluidsolid two-phase media. ${ }^{24)}$ It was reported that their proposed algorithm could succeed in (1) getting flaw images with better contrast, and (2) eliminating artifacts from multiple reflections of longitudinal waves in a transverse wave mode. However, the implementation method was still ambiguous in the robustness of applications. Further investigation on fluid-solid two phase media application was conducted by the authors, ${ }^{25)}$ proposing another efficient way to implement AWS into SAFT, namely AWS-SAFT. In this paper, AWS-SAFT is proposed for the imaging of flaws in a two solid media, which can be often applied to the inspection of infrastructure. In addition, ultrasonic beam radiations computed from AWS can be used to predict the Effective Region (ER) where high imaging capability can be anticipated.

In the following sections, the fundamentals and algorithms of SAFT and AWS-SAFT are explained. Then the performance of AWS-SAFT is experimentally tested for the imaging of a side-drilled hole (SDH) inside a steel plate. To improve the quality of flaw imaging, SSIM-MAP, which indicates the difference between the image of AWS-SAFT and the reference image for a specimen with no flaw, is introduced. The ER technique in conjunction with SSIM-MAP is proposed and verified using flaw images of an SDH at various positions. As a case study, a simplified model based on the fracture accident at Kisogawa Bridge is used to demonstrate the application of the ER technique.

\section{ULTRASONIC ANGLE BEAM TEST- ING USING ARRAY TRANSDUCER}

Ultrasonic angle beam testing is performed using a contact array transducer as shown in Fig. 1, where ultrasonic waves propagate into a steel plate through a polystyrene (PS) wedge placed beneath an array transducer.

The target for inspection is an SDH or a bottom flaw in a steel plate with $21.5 \mathrm{~mm}$ depth. In this paper, the cross-sections of an SDH and a bottom flaw are constant along the width of the steel plate. Therefore, the SAFT imaging can be performed in a twodimensional configuration.

The array transducer has 32 piezoelectric elements with the main frequency of $5 \mathrm{MHz}$ produced by KGK

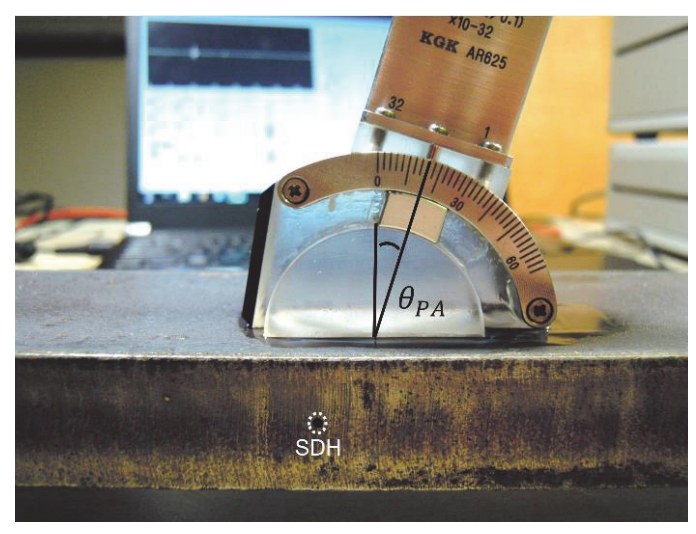

Fig.1 Ultrasonic angle beam test using array transducer. 
(Kanagawa, Japan). Each element size is $0.4 \mathrm{~mm} \times$ $10.0 \mathrm{~mm}$, and is aligned with the pitch of $0.5 \mathrm{~mm}$. The array transducer is put on the angle-adjustable PS wedge for the range of $\theta_{\mathrm{PA}}=0 \sim 60^{\circ}$.

The pulser-receiver used is JPR-600C produced by Japan Probe (Kanagawa, Japan), which gives a sinus oidal pulse with the main frequency of $5 \mathrm{MHz}$. Waveforms are acquired for all combinations of any two array elements. Hence, 32 times 32 waveforms are recorded for imaging of a flaw, when the array transducer with 32 elements is used. The sampling frequency is $50 \mathrm{MHz}$ with 16 times averaging to get signals stabilized. No gain is set in the amplifier in order to suppress noises to a minimum level.

Note that the wave velocities of longitudinal and transverse modes (L- and T-mode) in PS are 2300 $\mathrm{m} / \mathrm{s}$ and $1140 \mathrm{~m} / \mathrm{s}$, respectively, whereas the L- and T-wave velocities in steel are $5938 \mathrm{~m} / \mathrm{s}$ and $3240 \mathrm{~m} / \mathrm{s}$, respectively.

\section{SYNTHETIC APERTURE FOCUSING TECHNIQUE IMPLEMENTING THE APPROXIMATE WAVE SOLUTION (AWS)}

This section briefly explains the fundamentals and algorithms of $\mathrm{SAFT}^{8)}$ and AWS-SAFT. Assume that the ultrasonic wave propagating in the PS wedge is a longitudinal mode, and the wave in the steel plate is either a longitudinal or transverse mode (L-mode or T-mode).

\section{(1) Synthetic Aperture Focusing Technique (SAFT)}

A designated cross-sectional area for imaging a target object is defined as Region of Interest (ROI) which is discretized into pixels represented by the vector $\boldsymbol{b}=\left\{b_{1}, b_{2}\right\}$, similar to the production of a digital image. ${ }^{26)}$ Let $\boldsymbol{a}_{\boldsymbol{i}}$ and $\boldsymbol{c}_{\boldsymbol{j}}$ be the positions of a transmitter and a receiver, respectively. Referring to Fig. 2, the Time-of-Flight (TOF), $T O F_{a i, c j}^{\gamma}(\boldsymbol{b})$, for a specified wave propagating route from $\boldsymbol{a}_{\boldsymbol{i}}$ to $\boldsymbol{b}$ and from $\boldsymbol{b}$ to $c_{j}$ can be computed as:

$$
\operatorname{TOF}_{\boldsymbol{a} i, c j}^{\gamma}(\boldsymbol{b})=\frac{r_{i, 1}^{L}+r_{j, 1}^{L}}{c_{L, 1}}+\frac{r_{i, 2}^{\gamma}+r_{j, 2}^{\gamma}}{c_{\gamma, 2}}
$$

where the subscript $\gamma(=\mathrm{L}$ or $\mathrm{T})$ represents the wave mode in the steel plate; $c_{\gamma, \delta}$ represents the wave velocity of the wave mode $\gamma$ in the $\delta$-th solid medium where $\delta=1$ (PS wedge) and 2 (target); $r_{i, \delta}^{\gamma}$ and $r_{j, \delta}^{\gamma}$ indicate the travel distance of the $\gamma$-wave in the $\delta$-th medium for the transmitting route $(i)$ and the receiving route $(j)$, respectively. Refraction points $\boldsymbol{x}_{\boldsymbol{i}}$ and $\boldsymbol{x}_{\boldsymbol{j}}$ on the interface are determined numerically by a bisection method (with admissible error of $10^{-3} \mathrm{~mm}$ )

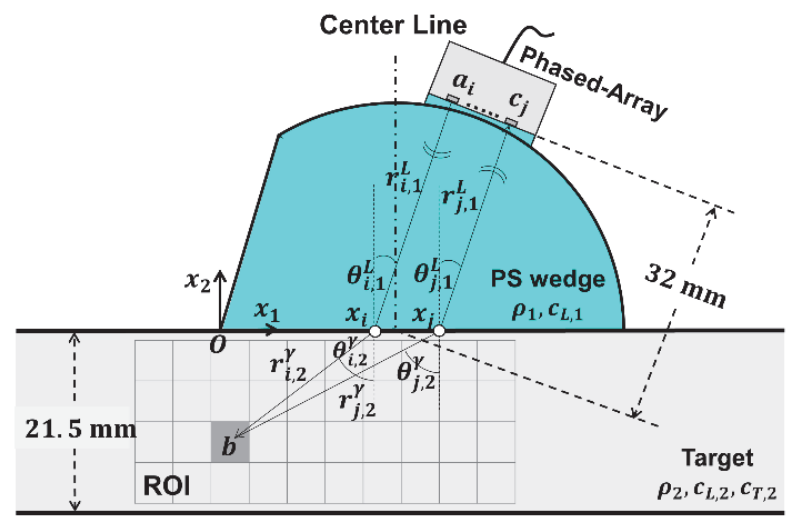

Fig.2 Schematic view of ultrasonic angle beam testing using array transducer.

on assumption that the refraction points are located between $\boldsymbol{a}_{\boldsymbol{i}}$ and $\boldsymbol{b}$ in the transmitting route, and $\boldsymbol{b}$ and $\boldsymbol{c}_{j}$ in the receiving route.

Let $A_{a i, c j}(t)$ be the waveform as a function of time $t$ for the combination of the transmitter $\boldsymbol{a}_{i}$ and the receiver $\boldsymbol{c}_{\boldsymbol{j}}$. If a point scatterer is located at point $\boldsymbol{b}$, a high scattered amplitude is expected to be observed as $\left|A_{a i, c j}\left(T O F_{a i, c j}^{\gamma}(\boldsymbol{b})\right)\right|$. Then the summation from all available pairs of array elements for transmitter and receiver is performed for the pixel point $\boldsymbol{b}$ as follows:

$$
S^{\gamma}(\boldsymbol{b})=\sum_{i} \sum_{j}\left|A_{a i, c j}\left(T O F_{a i, c j}^{\gamma}(\boldsymbol{b})\right)\right| .
$$

The location of a scatterer can be found from the distribution of $S^{\gamma}(\boldsymbol{b})$, since high amplitudes from a true scatterer are repeatedly superposed by the summation in Eq. (2). Therefore, plotting $S^{\gamma}(\boldsymbol{b})$ as a function of $\boldsymbol{b}$ in ROI will result in SAFT image of a scatterer.

\section{(2) Approximate Wave Solution (AWS) for ultra- sonic beam radiation}

An explicit solution of displacement field in a target material, generated by a planar array element, and transmitting through the interface between two solid media, can be approximated by various methods. ${ }^{27)-29)}$ In this paper, AWS based on a surface integral model derived by Schmerr ${ }^{23)}$ is used to improve the quality of flaw images by SAFT.

For the configuration shown in Fig. 2, the displacement of the $\gamma$ wave mode at point $\boldsymbol{b}$ due to the steadystate excitation of the array element at $\boldsymbol{a}_{\boldsymbol{i}}$ is expressed as

$$
\begin{aligned}
& u_{i}^{\gamma}(\boldsymbol{b}, \omega)=\frac{p_{0}}{2 \pi \rho_{1} c_{L, 1}^{2}} \\
& \int_{S_{T, a}} \frac{K_{\gamma}\left(\theta_{i, 1}^{L}\right) T_{12}^{\gamma, L} \exp \left(\mathrm{i} k_{L, 1} r_{i, 1}^{L}+\mathrm{i} k_{\gamma, 2} r_{i, 2}^{\gamma}\right)}{\sqrt{\Delta_{x}^{\gamma ; L}} \sqrt{\Delta_{y}^{\gamma ; L}}} d S\left(a_{i}\right)
\end{aligned}
$$


where $p_{0}$ is a uniform pressure acting on the array element surface, $\rho_{\delta}$ is the density of solid $\delta, k_{\gamma, \delta}$ represents the wave number for wave mode $\gamma$ of $\delta$-th solid, and $\mathrm{i}=\sqrt{-1}$. Other coefficients and functions required for the computation of AWS are shown in APPENDIX A. The integration is performed over the surface of element $a$, denoted as $S_{T, a}$.

For the receiving route, AWS is described in the same manner as the transmitting route, but the transmission coefficient $T_{12}^{\gamma ; L}$ must be changed to $T_{21}^{L ; \gamma}$ which can be calculated from Eq. (A.10) and the integration is performed over the surface of element $c$, denoted as $S_{T, c}$. Therefore, AWS for the receiving route shown in Fig. 2 can be written as Eq. (4).

$$
\begin{aligned}
& u_{j}^{\gamma}(\boldsymbol{b}, \omega)=\frac{p_{0}}{2 \pi \rho_{1} c_{L, 1}^{2}} \\
& \int_{S_{T, c}} \frac{K_{\gamma}\left(\theta_{j, 1}^{L}\right) T_{21}^{L, \gamma} \exp \left(\mathrm{i} k_{L, 1} r_{j, 1}^{L}+\mathrm{i} k_{\gamma, 2} r_{j, 2}^{\gamma}\right)}{\sqrt{\Delta_{x}^{\gamma ; L}} \sqrt{\Delta_{y}^{\gamma ; L}}} d S\left(c_{j}\right) .
\end{aligned}
$$

The integrations in Eqs. (3) and (4) are done numerically using a 5-point Gaussian integration.

It is well known that the accuracy of AWS is low at off-axis points, which are not located off the central axis of a main beam. However, since AWS-SAFT consisting of the amplitude of AWS at off-axis is smaller than that at on-axis, the AWS-SAFT proposed later is not much affected by the AWS at off-axis with low accuracy.

Fig. 3 shows displacement amplitudes of transmitted L-mode in the steel, calculated by AWS given in Eq. (3), when only the central array element at $a_{16}$ is excited by the pressure $p_{0}$ with the frequency 5MHz. Fig. 3(a) and (b) show the results in the cases of $\theta_{\mathrm{PA}}=0^{\circ}$ and $16^{\circ}$, respectively, where the angle $\theta_{\mathrm{PA}}$ represents an oblique incident angle to the surface of a steel plate. In Fig. 3 and the subsequent figures, the magnitude of displacement is expressed as the normalized value $|\boldsymbol{u}|\left(\rho_{1} c_{L, 1}^{2}\right) /\left(a p_{0}\right)$, where $a$ is the width of the array element.

As shown in Fig. 3, it is noted that relatively strong L-mode waves propagate from the array element of no. 16 into the steel plate in the direction normal to the nearest interface. Even for the angle $\theta_{\mathrm{PA}}$ of $16^{\circ}$, the strong beam radiation region appears in the steel just under the element no.16, because an ultrasonic beam emitted from the element is spread with certain angle width, and the beam amplitude in steel is determined by not only the incident beam amplitude, but also by the transmission coefficient.

Fig. 4 is the same as Fig. 3, except for the displacement amplitudes of the transmitted T-mode. Considering the Snell 's laws, the transverse wave resulting from mode conversion of the longitudinal wave striking at an incident angle $16^{\circ}$ should strongly propa-

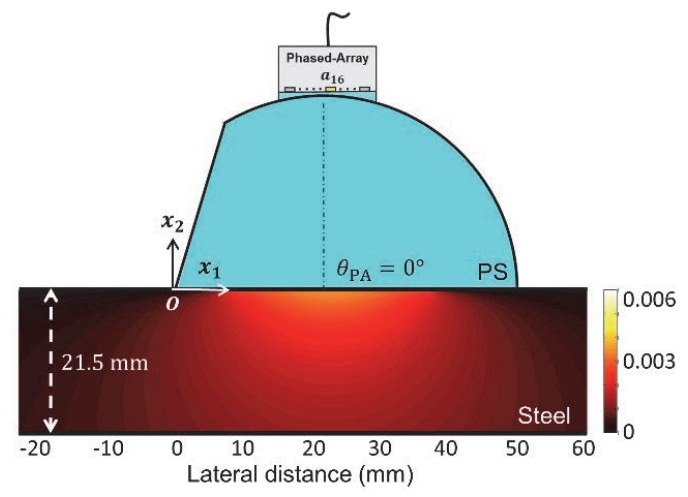

(a)

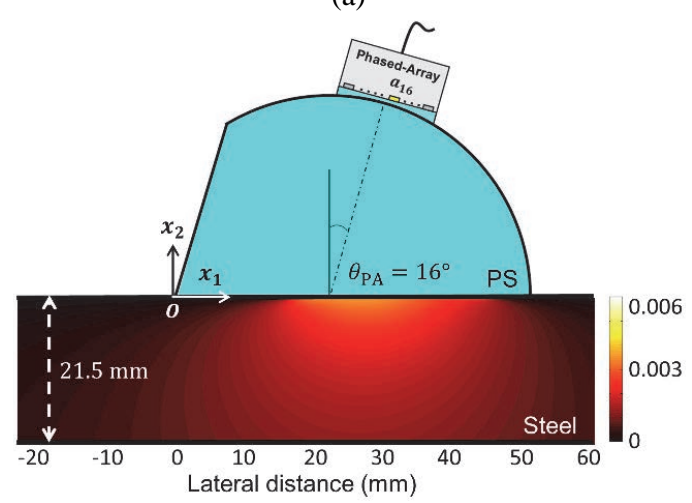

(b)

Fig.3 Beam radiation of L-mode in a steel plate, due to the array element no.16; (a) $\theta_{\mathrm{PA}}=0^{\circ}$ and (b) $\theta_{\mathrm{PA}}=16^{\circ}$.

gate in the direction of $23^{\circ}$ left from the normal to the interface. However, the transmission coefficient of mode converted transverse wave in steel, which can be computed from Eqs. (A.4)-(A.7), shows higher magnitude for the transverse wave converted from the longitudinal wave with the incident angle larger than the critical angle of $23^{\circ}$. As a result, the transverse wave mode refracted on the interface shows strong amplitude in $\pm 45^{\circ}$ directions from the normal of the interface as shown in Fig. 4. When $\theta_{\mathrm{PA}}=16^{\circ}$ as shown in Fig. 4(b), the region with strong beam radiation also shifts to the right, and the high intensity region in the forward direction becomes wider. Taking account of these beam radiations into SAFT imaging, pixel values will be amplified in the region with high intensity of AWS, and be suppressed in the other region.

\section{(3) Implementation of Approximate Wave Solu- tion (AWS) into Synthetic Aperture Focusing Technique (SAFT)}

As seen in Fig. 2, the pixel value at point $\boldsymbol{b}$ located in ROI for a pair of transmitter $i$ and receiver $j$ can be computed by $\left|A_{a i, c j}\left(T O F_{a i, c j}^{\gamma}(\boldsymbol{b})\right)\right|$. In principle, however, there are other pixel points $\boldsymbol{x}$ in ROI, for which the time of flight $T O F_{a i, c j}^{\gamma}(x)$ is the same value 


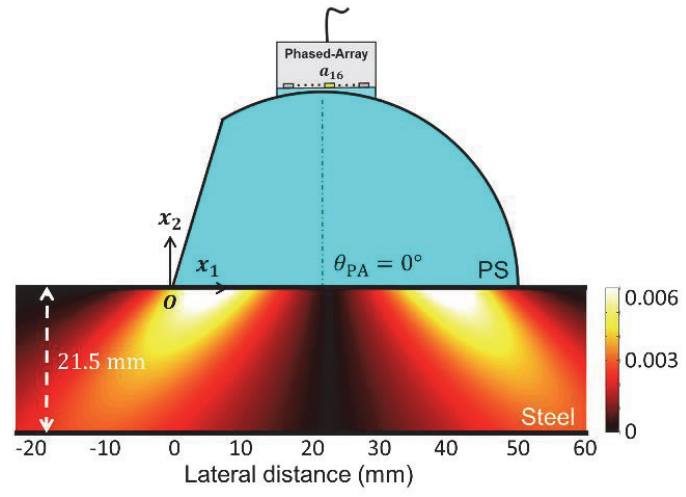

(a)

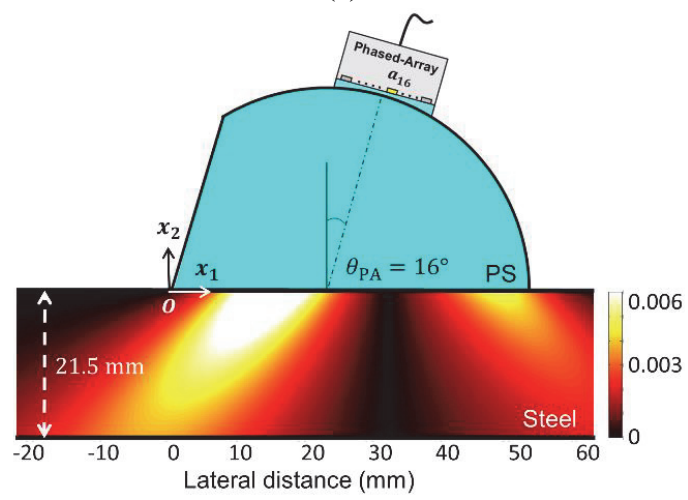

(b)

Fig.4 Beam radiation of T-mode in a steel plate, due to the array element no.16; (a) $\theta_{\mathrm{PA}}=0^{\circ}$ and (b) $\theta_{\mathrm{PA}}=16^{\circ}$.

as $T O F_{a i, c j}^{\gamma}(\boldsymbol{b})$. It means that when $S^{\gamma}(\boldsymbol{b})$ defined by Eq. (2) is plotted as a function of $\boldsymbol{b}$ in SAFT, the value of $\left|A_{a i, c j}\left(T O F_{a i, c j}^{\gamma}(\boldsymbol{b})\right)\right|$ can also be distributed as undesired noises even at other pixel points where transmitted waves are very weak or no real scatterer exists. That is why images created by a conventional SAFT sometimes become unclear.

As described in the previous section, displacement amplitudes at pixel points in ROI can be easily calculated by using AWS. It is, therefore, expected that the AWS can be used as a weight function in the SAFT imaging to reduce undesired noises at pixel points where no significant wave field exist. Hence we propose an improved formula of AWS-SAFT as

$$
A W S^{\gamma}(\boldsymbol{b})=\sum_{i} \sum_{j}\left|A_{\boldsymbol{a i}, \boldsymbol{c j}}\left(T O F_{\boldsymbol{a}, \boldsymbol{c} \boldsymbol{j}}^{\gamma}(\boldsymbol{b})\right) u_{i}^{\gamma}(\boldsymbol{b}) u_{j}^{\gamma}(\boldsymbol{b})\right| \text {. }
$$

To show the efficiency of the improved formula (5) of AWS-SAFT, we consider a test configuration as shown in Fig. 5 where $\theta_{\mathrm{PA}}$ is set to $16^{\circ}$ and $38^{\circ}$ for L- and T-modes, respectively. A side-drilled hole (SDH) with the diameter of $1.5 \mathrm{~mm}$ is processed into the steel plate of $21.5 \mathrm{~mm}$ thickness. Now we consider a single waveform obtained by the pitch-catch ultrasonic test using the 8th array element as a transmitter and the 24 th array element as a receiver. On

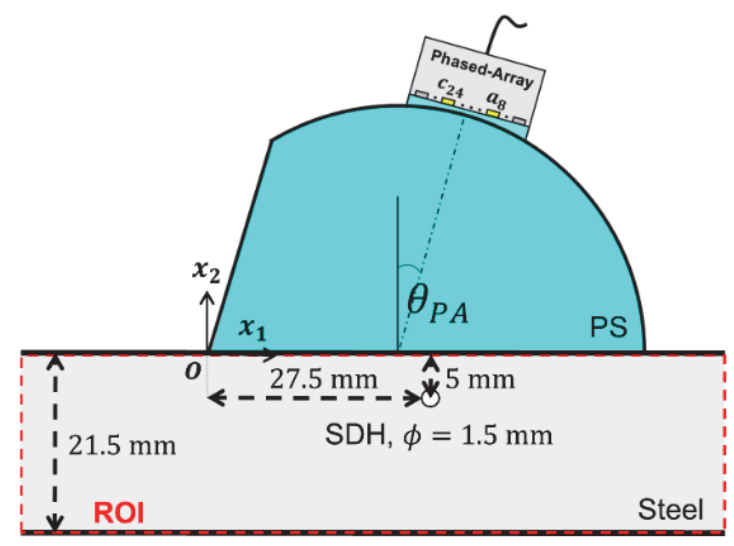

Fig.5 Test configuration for SDH.

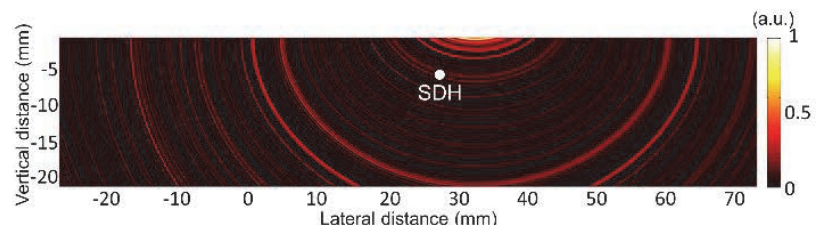

(a)

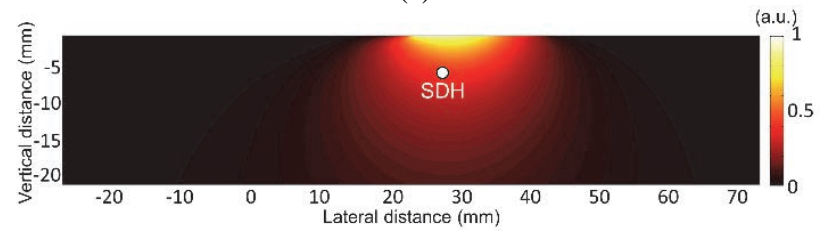

(b)

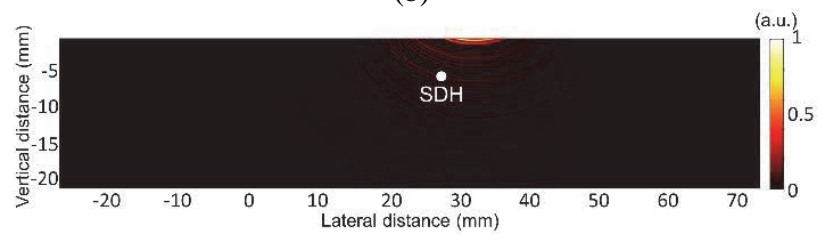

(c)

Fig.6 Image distribution of (a)

$$
\begin{aligned}
& \left|A_{\boldsymbol{a} 8, \boldsymbol{c} 24}\left(\operatorname{TOF}_{\boldsymbol{a} 8, \boldsymbol{c} 24}^{L}\right)(\boldsymbol{b})\right|, \quad \text { (b) }\left|u_{8}^{L}(\boldsymbol{b}) u_{24}^{L}(\boldsymbol{b})\right| \text { and } \\
& \text { (c) }\left|A_{\boldsymbol{a} 8, \boldsymbol{c} 24}\left(T O F_{\boldsymbol{a} 8, \boldsymbol{c} 24}^{L}(\boldsymbol{b})\right) u_{8}^{L}(\boldsymbol{b}) u_{24}^{L}(\boldsymbol{b})\right| .
\end{aligned}
$$

the assumption that longitudinal waves propagate in the steel specimen, Fig. 6(a) shows the image of $\left|A_{\boldsymbol{a} 8, \boldsymbol{c} 24}\left(\operatorname{TOF}_{\boldsymbol{a} 8, \boldsymbol{c} 24}^{L}(\boldsymbol{b})\right)\right|$, which corresponds to a part of a conventional SAFT. In this figure, it is shown that the amplitudes of reflected longitudinal waves are distributed not only at the SDH but also in the side area of the SDH. Fig. 6(b) shows the displacement amplitudes of AWS, $\left|u_{8}^{L}(\boldsymbol{b}) u_{24}^{L}(\boldsymbol{b})\right|$, as a function of the position $\boldsymbol{b}$ in ROI. Fig. 6(c) illustrates the values of $\left|A_{\boldsymbol{a} 8, \boldsymbol{c} 24}\left(\operatorname{TOF}_{\boldsymbol{a} 8, \boldsymbol{c} 24}^{L}(\boldsymbol{b})\right) u_{8}^{L}(\boldsymbol{b}) u_{24}^{L}(\boldsymbol{b})\right|$, which is a part of AWS-SAFT. Compared with Fig. 6(a), Fig. 6(c) gives an image intensity more concentrated around the SDH. Fig. 7(a) to (c) are the same figures as Fig. 6(a) to (c), respectively, but assuming the propagation of transverse waves in a target specimen. The same 


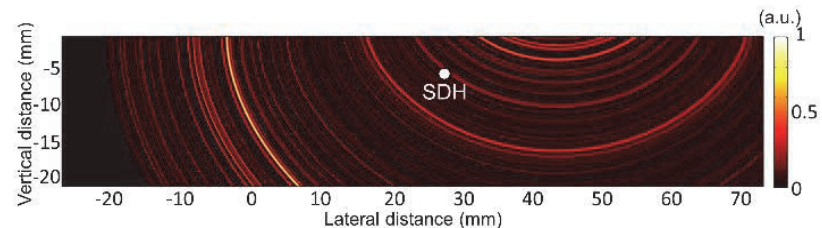

(a)

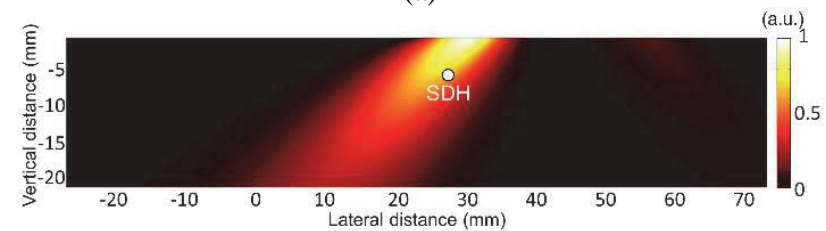

(b)

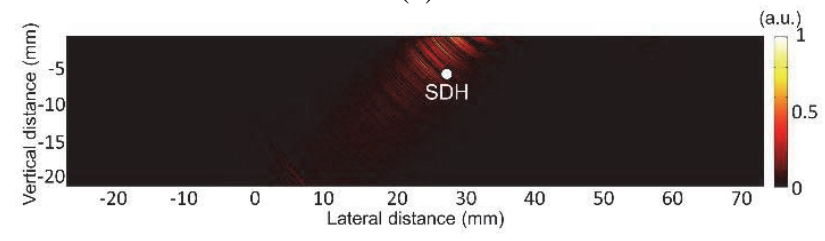

(c)

Fig.7 Image distribution $\left|A_{\boldsymbol{a} 8, \boldsymbol{c} 24}\left(\operatorname{TOF}_{\boldsymbol{a} 8, \boldsymbol{c} 24}^{T}\right)(\boldsymbol{b})\right|, \quad$ (b) $\quad\left|u_{8}^{T}(\boldsymbol{b}) u_{24}^{T}(\boldsymbol{b})\right| \quad$ and (c) $\left|A_{a 8, c 24}\left(T O F_{a 8, c 24}^{T}(\boldsymbol{b})\right) u_{8}^{T}(\boldsymbol{b}) u_{24}^{T}(\boldsymbol{b})\right|$.

results are obtained as in the case of Fig. 6.

From Fig. 6 and Fig. 7, it is expected that the image intensity $A W S^{\gamma}(\boldsymbol{b})$ of AWS-SAFT, given by Eq. (5), becomes clearer than that of the image of a conventional SAFT given by $S^{\gamma}(\boldsymbol{b})$ in Eq. (2). The efficiency of AWS-SAFT will be discussed in the next section.

\section{(4) Characteristics of the AWS-SAFT}

In this section, the ultrasound imaging by AWSSAFT is demonstrated and its characteristics are discussed using experimental results. The configuration of the experiment is the same as Fig. 5. Experimental results are shown in Fig. 8 and Fig. 9 for L- and Tmodes, respectively. In these figures, figures (a) and (b) show images obtained by the conventional SAFT and the AWS-SAFT, respectively; $\theta_{\mathrm{PA}}$ is set to $16^{\circ}$ and $38^{\circ}$ for L- and T-modes, respectively.

As shown in Fig. 8(a) and (b), image amplitudes are localized around the SDH by SAFT and AWS-SAFT, respectively. In the image of AWS-SAFT, noises in the region, where the intensity of ultrasonic beam radiation is low, are reduced; hence the SDH image of AWS-SAFT becomes clearer than that of the normal SAFT. However, the SDH image obtained by AWSSAFT still gets blurred, since the pixel values in the region near the top surface of the steel plate are highly amplified by the refracted wave.

In Fig. 9(a) and (b), images using T-modes also show local concentration near the SDH by SAFT and AWS-SAFT, respectively. In the SAFT image,

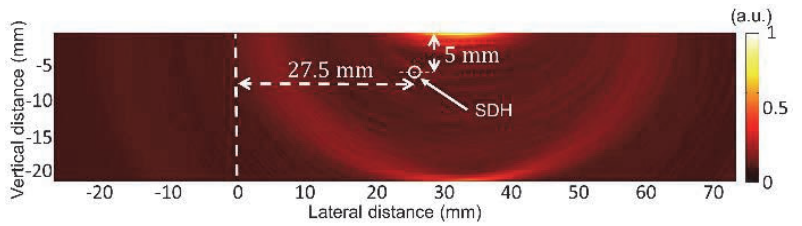

(a)

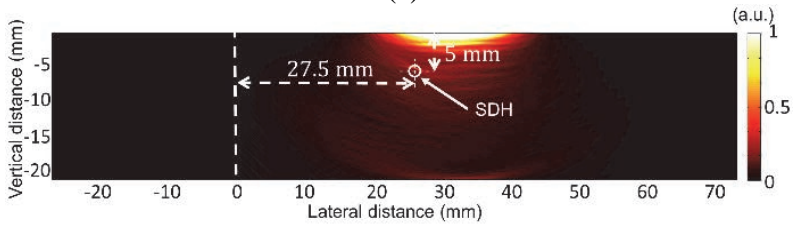

(b)

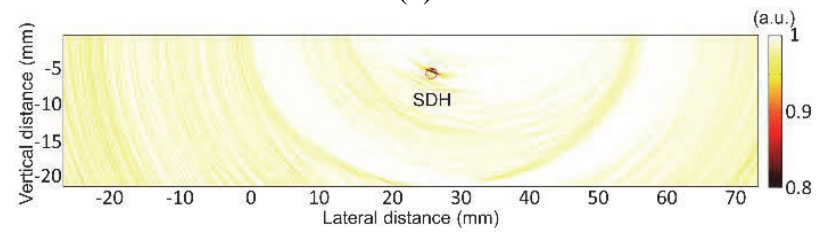

(c)

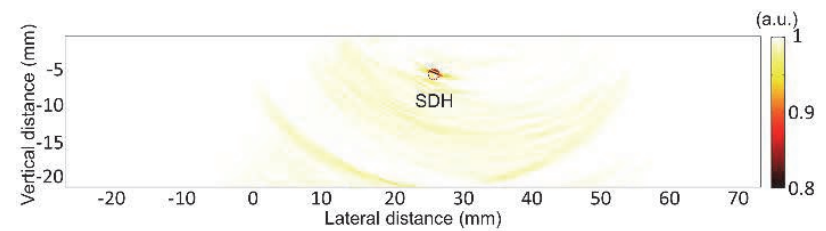

(d)

Fig.8 Flaw images obtained by (a) SAFT, (b) AWS-SAFT, (c) SSIM-MAP of SAFT, and (d) SSIM-MAP of AWS-SAFT, in L-mode.

however, artifacts due to longitudinal waves reflected from the bottom of the specimen and other parts are observed at various locations, which can lead an inspector to wrong evaluation of a flaw position. By using AWS-SAFT, the artifacts can be screened out as shown in Fig. 9(b). Similar to L-mode, additionally, noises in the region with low intensity of ultrasonic beam radiation are mostly suppressed. However, pixel values in the region closed to the top surface are still highly amplified.

As a summary, AWS-SAFT can improve SAFT images effectively by reducing overall noises and eliminating artifacts due to longitudinal waves in the Tmode image. It is noted that the computational time for the AWS part in AWS-SAFT is only $1 \%$ of the time for the SAFT part. However, AWS-SAFT images still get blurred for a flaw near the top surface due to large refracted waves. Therefore a more efficient technique is required to diminish the effect of refraction and other noises. For this purpose, we introduce here the use of Structural SIMilarity (SSIM) index ${ }^{31)}$ as a supporting aid in viewing the results. 


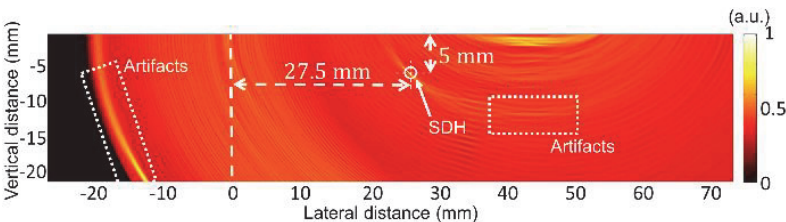

(a)

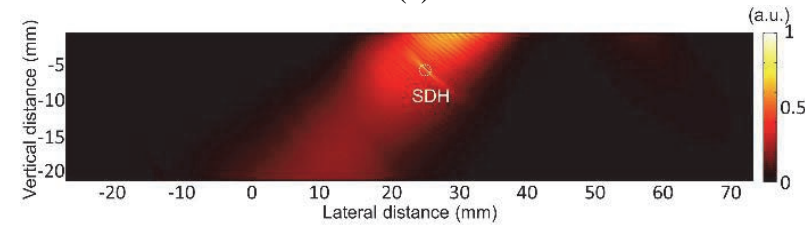

(b)

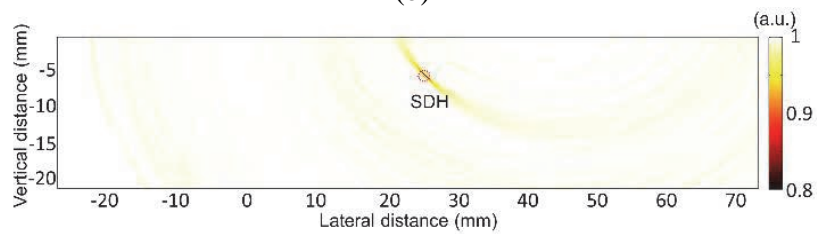

(c)

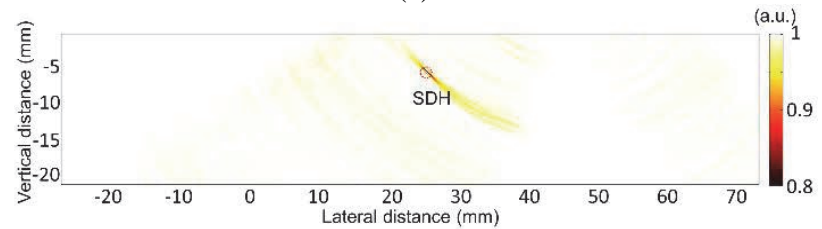

(d)

Fig.9 Flaw images obtained by (a) SAFT, (b) AWS-SAFT, (c) SSIM-MAP of SAFT, and (d) SSIM-MAP of AWS-SAFT, in T-mode.

\section{STRUCTURAL SIMILARITY INDEX (SSIM)}

As described in detail in APPENDIX B, SSIM is an index to identify the difference between two images. SSIM index can also be used to compare two images in detail, segment by segment. When the number of segments is equal to the number of pixels of images, the comparison is performed pixel by pixel. The values of SSIM are stored into a matrix with the same size as the image and are plotted as "SSIM-MAP." In this paper, this approach is applied to obtain a flaw image with high contrast.

To use this approach, the waveform data from a non-defective specimen are required as reference signal. SSIM is obtained from a pair of images constructed by flaw signals and reference ones to clearly boost a defect image. Fig. 8(c) and Fig. 9(c) show SSIM-MAPs for the contentional SAFT results of Land T-modes, respectively. Whereas, SSIM-MAPs for AWS-SAFT results of L- and T- modes are shown in Fig. 8(d) and Fig. 9(d), respectively. SDHs in all images using SSIM-MAP become very clear, hence the location of the flaw can be identified much easier. Comparing Fig. 8(c) and (d), SDH in the SSIMMAP from AWS-SAFT image contains less noises

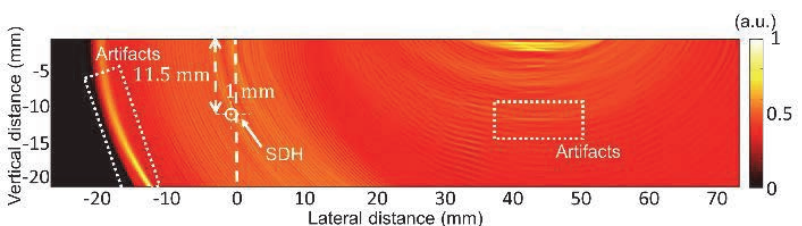

(a)

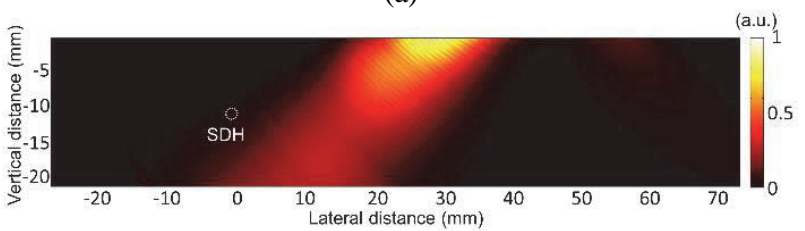

(b)

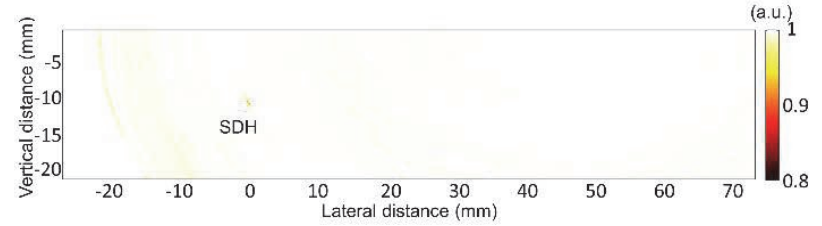

(c)

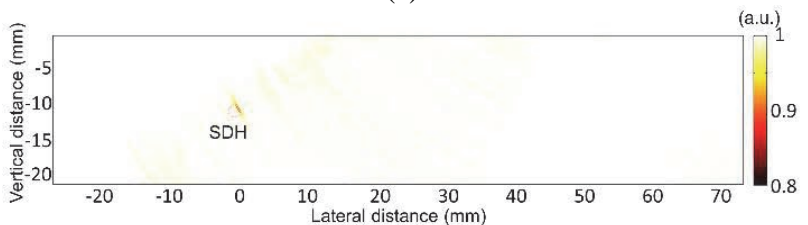

(d)

Fig.10 Flaw images obtained by (a) SAFT, (b) AWSSAFT, (c) SSIM-MAP of SAFT and (d) SSIMMAP of AWS-SAFT, in T-mode of an effective case.

than SSIM-MAP from the conventional SAFT. Comparing Fig. 9(c) and (d), SDHs in both images are detectable with almost the same contrast.

Fig. 10 is another example of the imaging of an SDH using T-mode to demonstrate the effectiveness of using SSIM-MAP. In the conventional SAFT and AWS-SAFT images of Fig. 10(a) and (b), the SDH cannot be recognized since the image intensity for the SDH is very weak comparable to noise. SSIMMAPs based on the conventional SAFT and AWSSAFT are shown in Fig. 10(c) and (d), respectively. Comparing both images, SDH image can be observed in Fig. 10(d), while it cannot be observed in Fig. 10(c). These results show that applying the SSIMMAP to AWS-SAFT can boost the image intensity better than the conventional SAFT.

Results of imaging hereafter will be shown using the SSIM-MAPs of AWS-SAFT results for flaw identification. According to our experiments, it is essential to be aware that changing the wedge angle can result in poor SSIM-MAP since the SSIM index is very sensitive. 


\section{EFFECTIVE REGION (ER) TECH- NIQUE}

\section{(1) Effective Region (ER)}

In ultrasonic imaging, results can be varied depending on probe position, frequency, power, and other test configuration. To quantitatively determine whether the current test configuration is covering the ROI, which is suspected to contain flaw or defect, Effective Region (ER) technique is proposed as a method to assess imaging capability in the ROI by using Approximate Beam Radiation (ABR) computed from AWS.

The intensity of ultrasonic beam radiation $u_{i}^{\gamma}(\boldsymbol{b}, \omega)$ in Eq. (3), which is considered an important factor for successful imaging, can be evaluated at point $\boldsymbol{b}$ due to the excitation of a single array element at $\boldsymbol{a}_{\boldsymbol{i}}$. To produce an ultrasound image using SAFT or AWSSAFT, however, all available elements of a phasedarray transducer are normally used. Therefore, it is necessary to predict the trend of ultrasonic beam radiation when all elements are used in transmitting ultrasonic waves. Based on this concept, ABR for the wave mode $\gamma$ is defined as $\sum_{i=1}^{n}\left|u_{i}^{\gamma}(\boldsymbol{b}, \omega)\right|$, where nondimensional displacements are used.

ABRs for a 32-channel phased-array transducer, which will be used throughout this paper, are shown in Fig. 11 and Fig. 12 for L-mode $\left(\theta_{\mathrm{PA}}=16^{\circ}\right)$ and Tmode $\left(\theta_{\mathrm{PA}}=38^{\circ}\right)$, respectively. These angles are chosen empirically since they provide high ABR intensities. Contour lines are marked with equally spaced intervals of 0.025 . Here, we make a hypothesis that in the region with higher ABR intensity, flaw imaging with high contrast can be obtained. In other words, if a flaw is located inside the region with higher ABR intensity, it is predicted that clearer image can be reconstructed for the flaw than for the one located inside

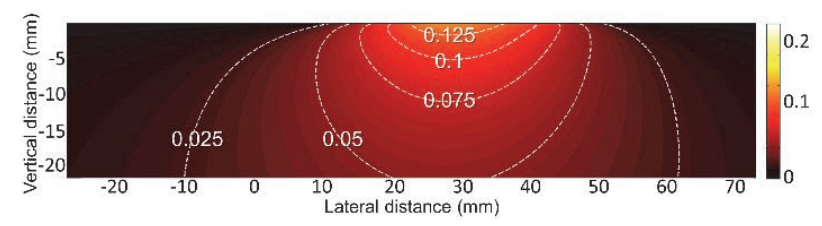

Fig.11 Distribution of ABR intensity of L-mode with $\theta_{\mathrm{PA}}=16^{\circ}$.

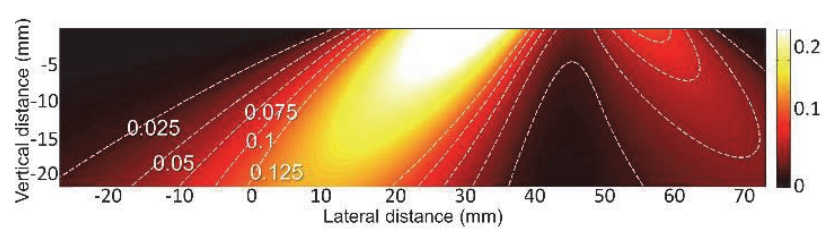

Fig.12 Distribution of ABR intensity of T-mode with $\theta_{\mathrm{PA}}=38^{\circ}$. the region with lower $A B R$ intensity.

Here, we define ER as a region with the ABR intensity over a certain threshold value, where a flaw can be imaged properly. In the following section, a proper threshold of ABR intensity is determined experimentally to find a reasonable ER.

\section{(2) Experimental verification of Effective Region (ER) Technique}

Experiments using the same angle beam ultrasonic equipment as shown in Fig. 5 are performed for imaging several SDHs located at different positions in a steel plate specimen. The position of an SDH in each case is shown in each figure. In the flaw imaging, SSIM-MAPs are reconstructed to find a common threshold value $\chi$ of $A B R$ intensity for both $L$ and $T$ modes.

Fig. 13-Fig. 15 show the results using L-mode with $\theta_{\mathrm{PA}}=16^{\circ}$ for SDHs at different positions of D1, D2, and D3. In these figures, (a)-(c) show the distribution of ABR intensity, the SSIM-MAP, and the magnified image of the SSIM-MAP around the SDH, respectively. Fig. 16-Fig. 19 show the same results as those in Fig. 13-Fig. 15, but use T-mode with $\theta_{\mathrm{PA}}=38^{\circ}$ for SDHs at different positions of D1T, D2T, D3T, and D4T.

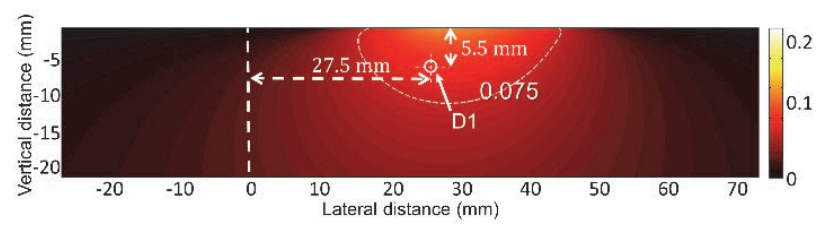

(a)

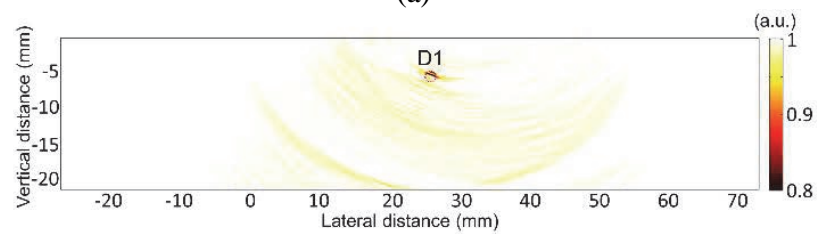

(b)

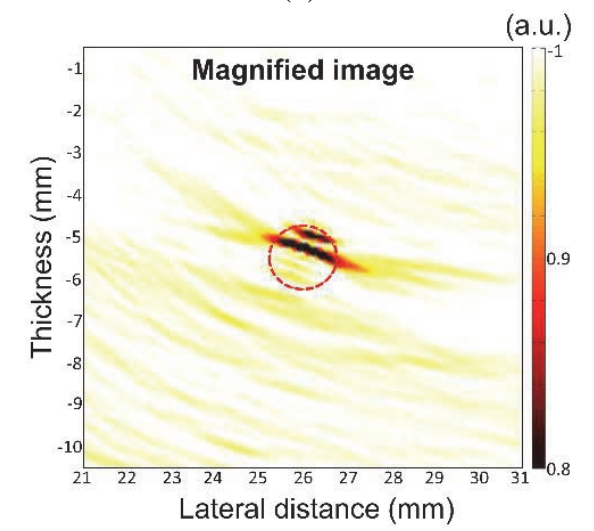

(c)

Fig.13 (a) ABR intensity, (b) SSIM-MAP, and (c) magnified SSIM-MAP of L-mode for the SDH of D1. 


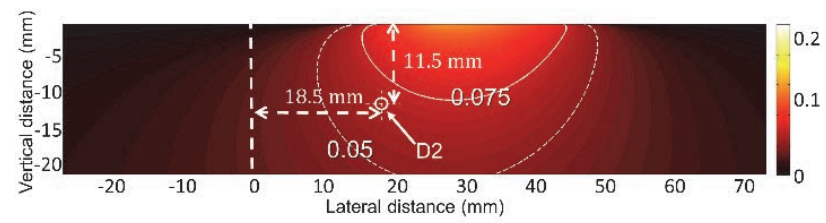

(a)

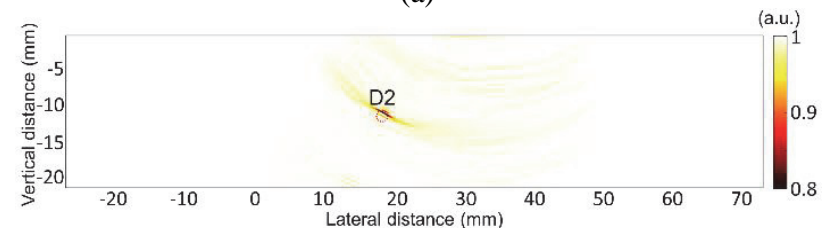

(b)

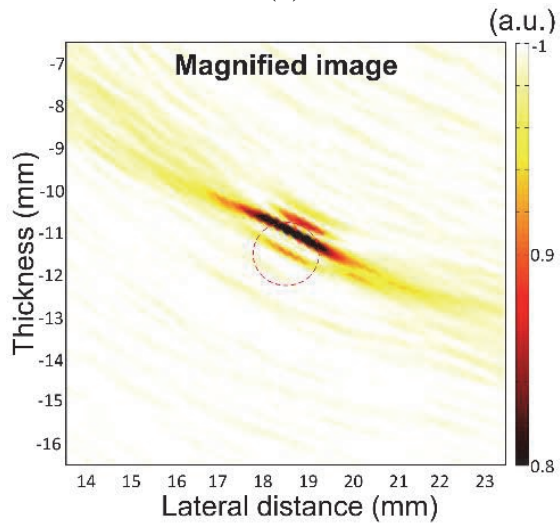

(c)

Fig.14 (a) ABR intensity, (b) SSIM-MAP, and (c) magnified SSIM-MAP of L-mode for the SDH of D2.

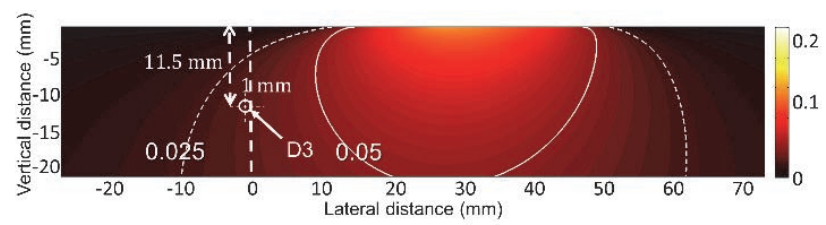

(a)

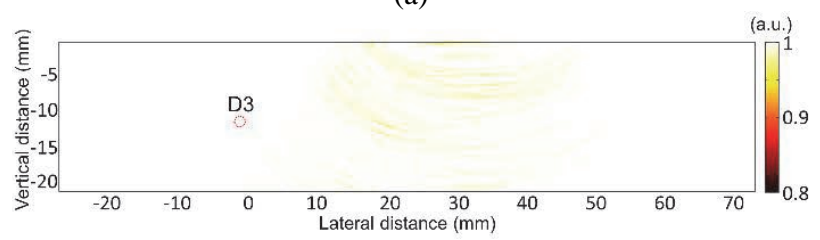

(b)

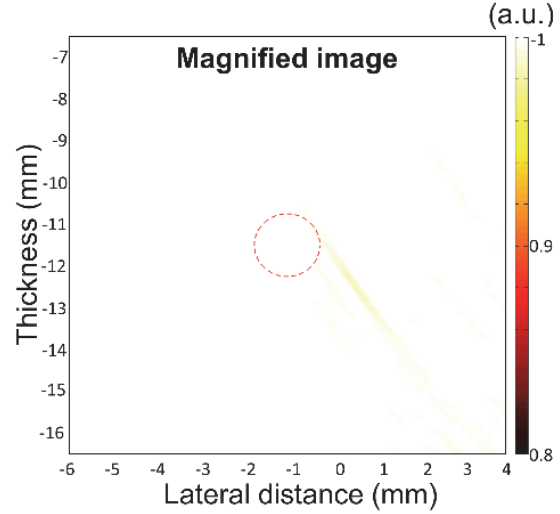

(c)

Fig.15 (a) ABR intensity, (b) SSIM-MAP, and (c) magnified SSIM-MAP of L-mode for the SDH of D3.

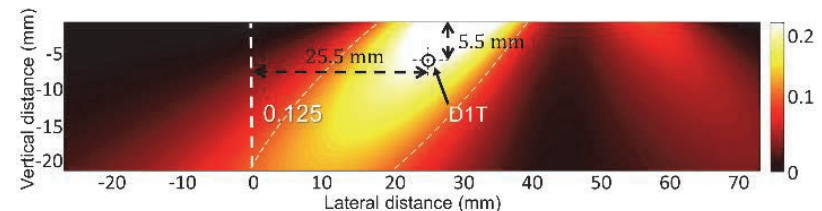

(a)

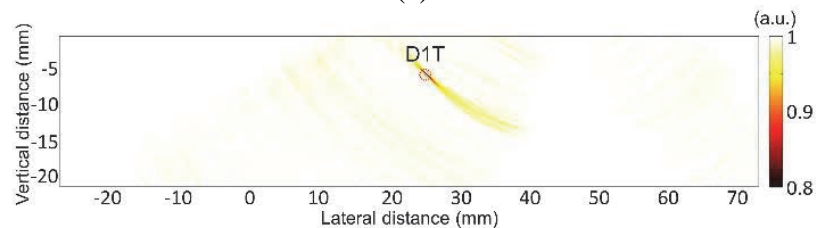

(b)

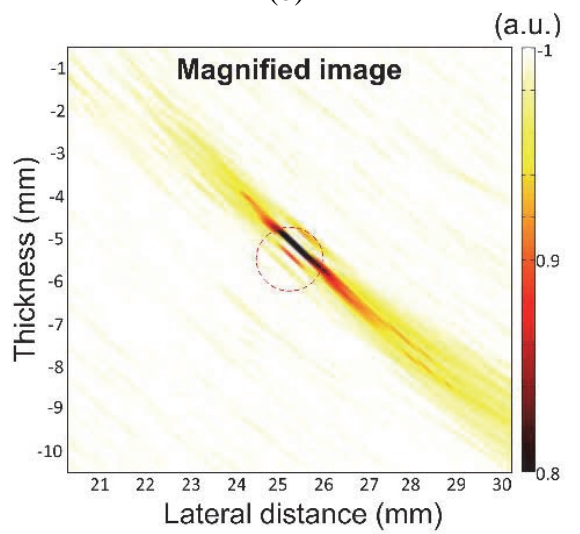

(c)

Fig.16 (a) ABR intensity, (b) SSIM-MAP, and (c) magnified SSIM-MAP of T-mode for the SDH of D1T.

As seen in Fig. 13 and Fig. 14, the SDHs of D1 and D2 are imaged well, whereas the SDH of D3 cannot be reconstructed properly as shown in Fig. 15. Hence, it can be implied that if a flaw is located in the region with the ABR value of L-mode over 0.05 , a flaw image can be reconstructed well. Therefore, the threshold $\chi$ of L-mode ABR is selected as $\chi=0.05$ to define a reasonable ER.

As for flaw imaging with T-mode, the SDHs of D1T, D2T, and D3T are imaged properly as seen in Fig. 16-Fig. 18. However, the SDH of D4T cannot be recognized even in the magnified image shown in Fig. 19(c). Thus, it can be said that ER is defined as the region with the ABR intensity more than 0.05 . Similar to L-mode, therefore, the same value of 0.05 is selected as the threshold $\chi$ for ER in T-mode.

According to experimental results for several SDHs in the steel plate, it can be implied that ERs for both $\mathrm{L}$ and T-modes are regions where the intensities of ABR are greater than 0.05 . For L-mode, the ER is the region underneath the transducer, whereas the ER for Tmode is the regions in approximately $\pm 45^{\circ}$ directions inclined to the top surface of the specimen. Although the shapes of ER for L- and T-modes are different, it is found that the threshold $\chi=0.05$ is common for ERs for both wave modes. 


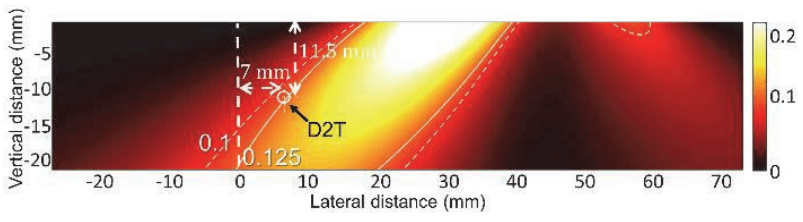

(a)

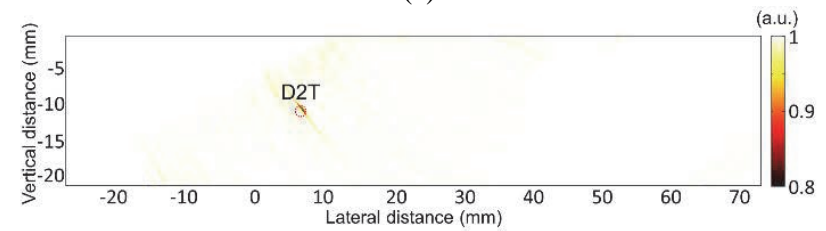

(b)

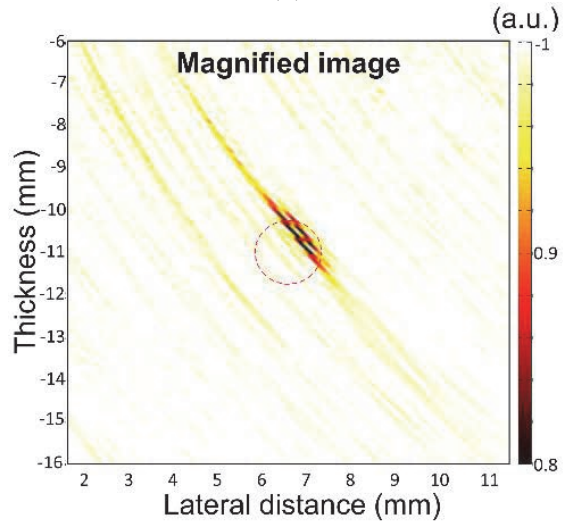

(c)

Fig.17 (a) ABR intensity, (b) SSIM-MAP, and (c) magnified SSIM-MAP of T-mode for the SDH of D2T.

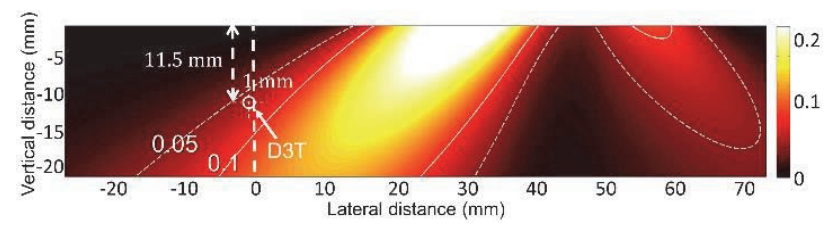

(a)

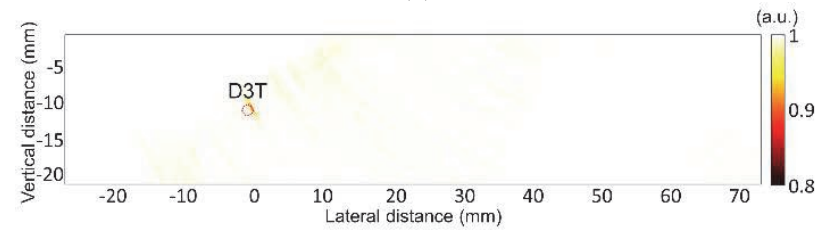

(b)

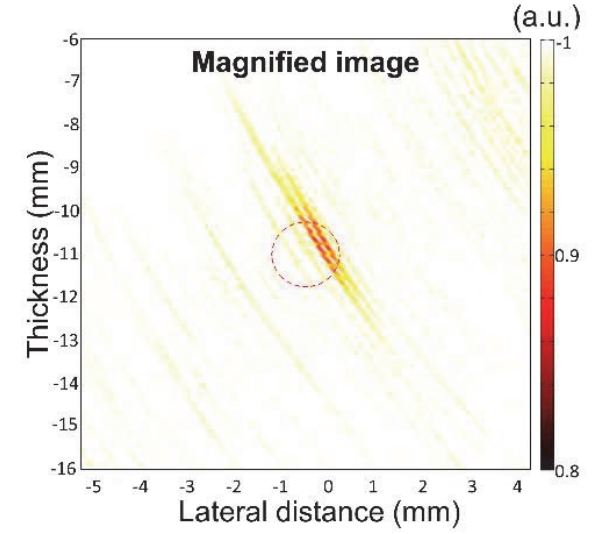

(c)

Fig.18 (a) ABR intensity, (b) SSIM-MAP, and (c) magnified SSIM-MAP of T-mode for the SDH of D3T.

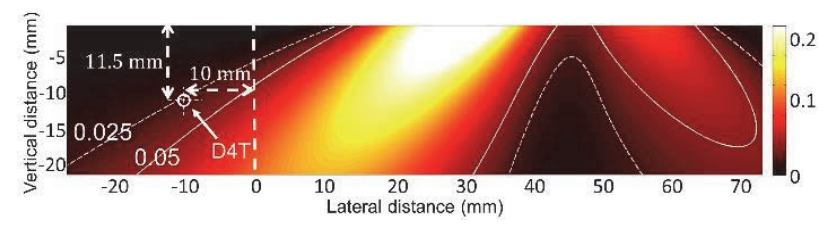

(a)

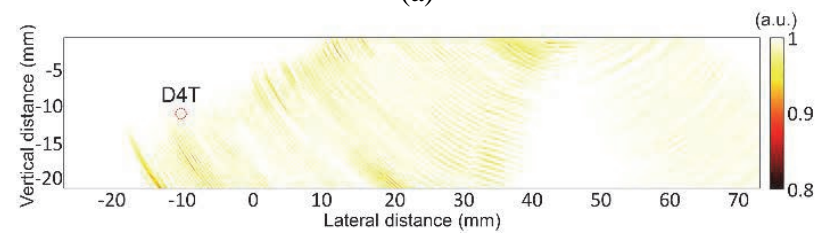

(b)

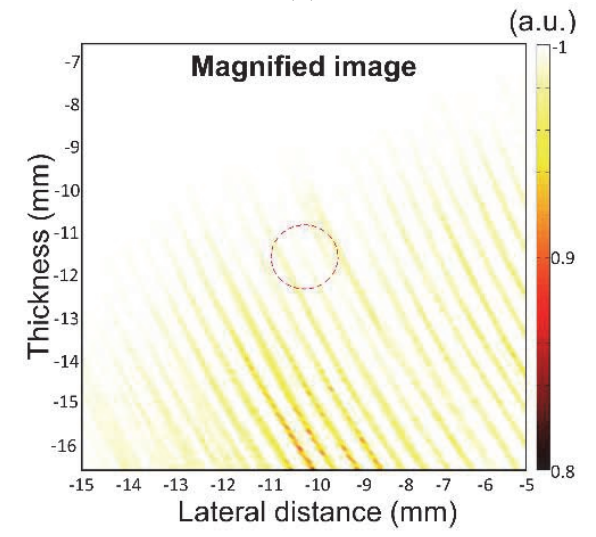

(c)

Fig.19 (a) ABR intensity, (b) SSIM-MAP, and (c) magnified SSIM-MAP of T-mode for the SDH of D4T.

\section{IMAGING OF BOTTOM DEFECT OF STEEL PLATE}

\section{(1) Outline of problem and experimental setup}

The accident of fracture at Kisogawa truss bridge occurred in June 2007. Corrosion of a steel truss member embedded into a concrete slab had been progressing, and it could not be detected until it fractured. This led to a need to develop a way for early detection of a flaw inside a region that is difficult to inspect with conventional NDT methods.

The AWS-SAFT and ER technique with SSIMMAP developed in the previous sections has potential to be useful for early detection of a flaw in a steel member embedded in concrete. However, it would be too complicated to attempt an actual threedimensional structural member from the beginning. Here, therefore, we use a simplified two-dimensional model for experiment as shown in the right side of Fig. 20, where the effect of concrete is imitated by setting an inspection limit. The parameter $L(>0)$ is defined as the distance from the front edge of a PS wedge to the right edge of a rectangular flaw. A long rectangular notch with the cross-section of $20 \mathrm{~mm} \times$ $5 \mathrm{~mm}$ is processed onto the steel plate bottom surface to imitate the thickness reduction resulting from cor- 


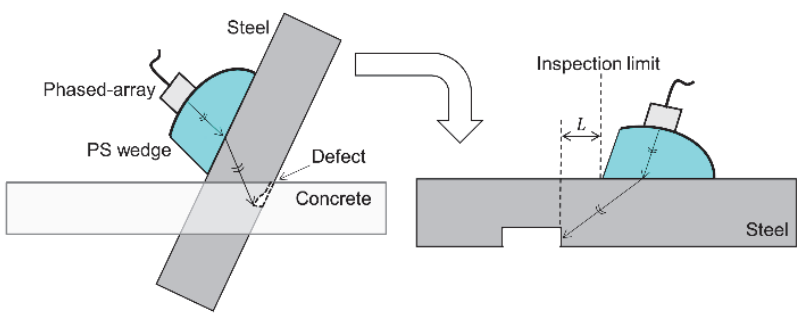

Fig.20 Simplified model for fractured truss member.

rosion.

Experiments of angle beam ultrasonic testing with an array transducer are performed for the rectangular notch on the bottom surface, apart from the transducer with different distances of $L=1,10 \mathrm{~mm}$, and $20 \mathrm{~mm}$. All devices and materials used in this section are the same as those in Section 2 and the setup of experiment in this section is shown in Fig. 20. The angle $\theta_{\mathrm{PA}}$ for the array transducer is fixed at $20^{\circ}$.

\section{(2) Experimental results}

Fig. 21(a) and (b) show ERs of L and T-modes, respectively, with the threshold of 0.05 for the rectangular notch located with the distance $L=1 \mathrm{~mm}$. As shown in Fig. 21(a), the rectangular notch, represented by a dashed line, is located outside the ER. It is, therefore, impossible to reconstruct the notch using L-mode in this configuration according to the ER technique. As shown in Fig. 21(b), on the other hand, the ER of T-mode covers the notch region. Therefore, T-mode is considered as an appropriate wave mode for the imaging of the notch.

SSIM-MAP based on the T-mode AWS-SAFT is shown in Fig. 21(c). For oblique incidence from the right-upper side, not only the corner diffraction but also the reflected waves by both the bottom surface and the vertical notch surface are considered as corner diffraction depicted in SAFT imaging. Therefore, the bottom-right corner of the notch is reconstructed very strongly. In addition to the corner diffraction, the top-right corner of defect can also be seen in the image. The magnified image is shown in Fig. 21(d), to show the image intensity of the top-right corner of defect. As the image of the top-right corner is reconstructed from weakly scattered waves from sharp rectangular edge, the image intensity is relatively low compared with the bottom-right corner; however, it is still high compared with the noises, hence it cannot be ignored. Moreover, the depth of notch can be determined using the distance between the top-right and bottom-right corners.

Fig. 22(a) and (b) are the same as Fig. 21 (b) and (c), respectively, but for the case of $L=10 \mathrm{~mm}$. As shown in Fig. 22(a), the right part of the notch is still inside the ER, and thus it shall be reconstructed

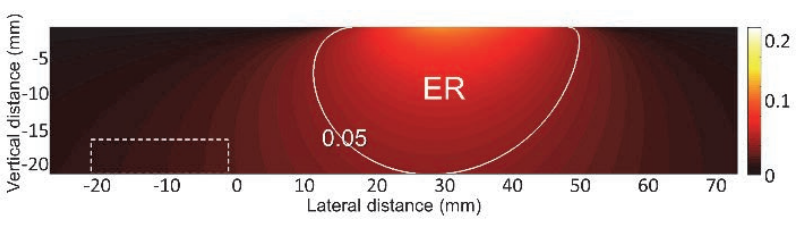

(a)

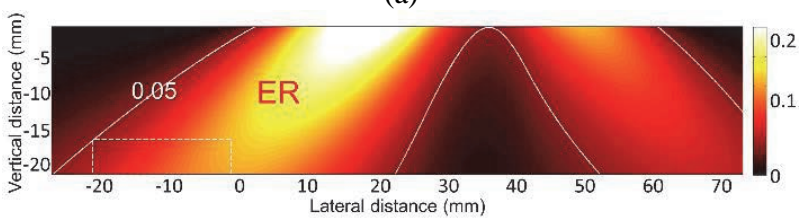

(b)

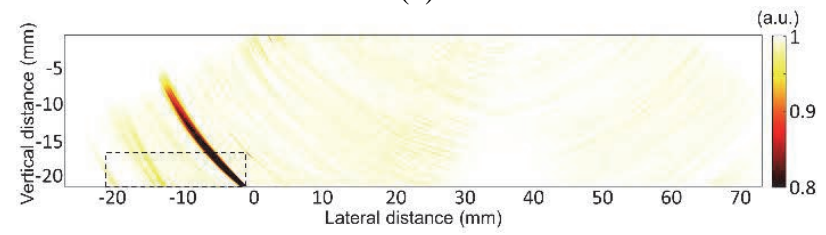

(c)

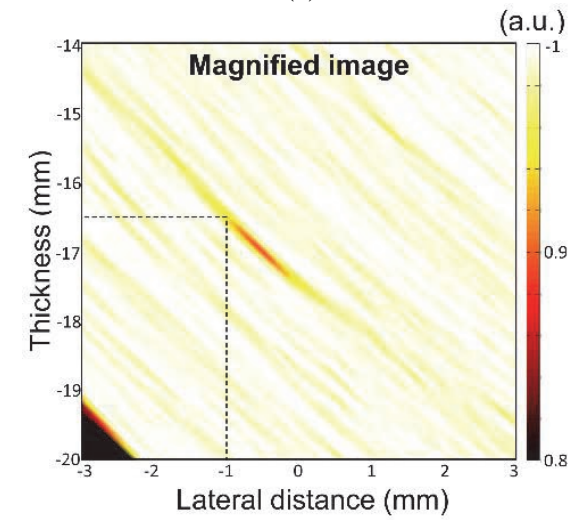

(d)

Fig.21 (a) ER of L-mode, (b) ER of T-mode, (c) SSIMMAP, and (d) magnified SSIM-MAP of T-mode for rectangular notch located with $L=1 \mathrm{~mm}$.

clearly. The experimental result is shown in Fig. 22(b). The bottom-right corner of the defect can be detected clearly. In this configuration, the top-right edge of defect cannot be reconstructed, so the depth of notch cannot be determined. However, it still proves the effectiveness of the ER technique in checking the region inside ROI in which a flaw image can be reconstructed definitely.

Fig. 23 is the same as Fig. 22, but for $L=20 \mathrm{~mm}$. In this case, the bottom-right corner of the notch is on the border of the ER of T-mode as shown in Fig. 23(a). SSIM-MAP based on the T-mode is shown in Fig. 23(b). Although the bottom-right corner is reconstructed to be recognized, the intensity of the image is much lower than in the cases of $L=1$ and $10 \mathrm{~mm}$.

As seen in Fig. 21-Fig. 23, the flaw imaging based on SSIM-MAP has good correlation with the ER defined by the threshold of ABR intensity. Hence it shows that the ER technique is very effective to estimate a high imaging capability region before testing. 


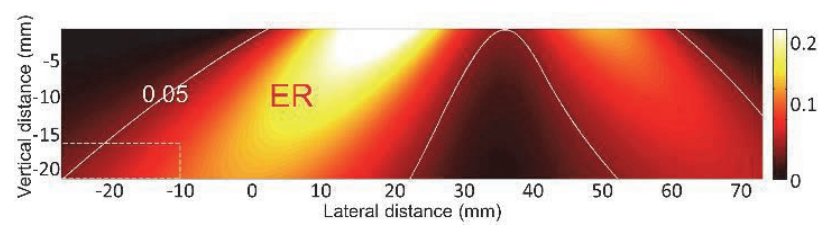

(a)

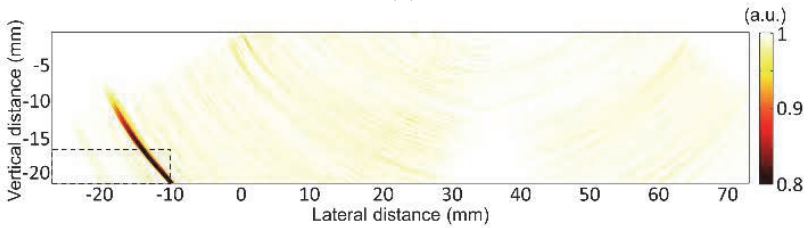

(b)

Fig.22 (a) ER of T-mode and (b) SSIM-MAP of T-mode for rectangular notch located with $L=10 \mathrm{~mm}$.

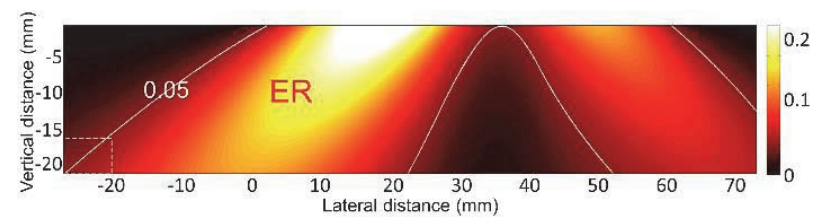

(a)

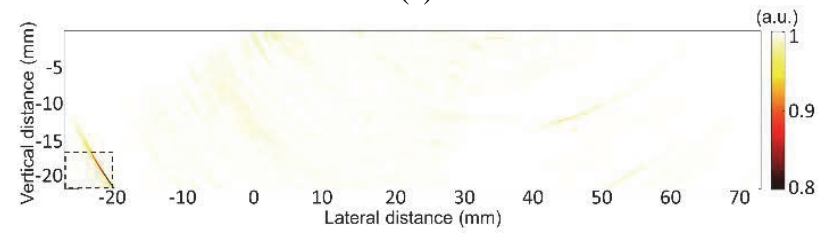

(b)

Fig.23 (a) ER of T-mode and (b) SSIM-MAP of T-mode for rectangular notch located with $L=20 \mathrm{~mm}$.

\section{CONCLUSION}

AWS-SAFT was proposed as an improved version of conventional SAFT by implementing ultrasonic beam radiation, approximated using the AWS. This paper focused on the performance of AWS-SAFT in solid-solid angle beam testing. AWS-SAFT has the following improvements:

(1) Artifacts due to bottom echoes and multiple reflections of longitudinal waves can be eliminated in T-mode results.

(2) Flaw images in AWS-SAFT are more distinct from background noises than those in SAFT because noises in regions outside the ER are suppressed.

However, AWS-SAFT images still get blurred for flaws near the top surface due to large refracted waves. SSIM-MAP was proposed as a supporting aid for easier identification of the flaw.

Furthermore, ER technique was proposed as a method to assess imaging capability in the ROI by using ABR computed from AWS. This technique was experimentally tested and it was found that the region in either L- or T-mode where ABR intensities are over 0.05 can be defined as the ER, for which image capability is remarkably high. The ER for L-mode is the region underneath the transducer, whereas for Tmode, it is the inclined region approximately $\pm 45^{\circ}$ directions. The ER technique is very useful in helping engineers and inspectors to select an appropriated transducer for any arbitrary test situation.

Finally, the use of ER technique was demonstrated for imaging of a steel bottom defect with the restrained condition of an inspection limit. Imaging capability can be well predicted using the ER technique.

In this paper, the AWS-SAFT and ER techniques are applied to simplified 2D problems only. However, since AWS for a 3D configuration is available, ${ }^{20)}$ our proposed technique will be further applied to various configurations, especially for: (1) 3-D flaws in a complex structure, and (2) defects buried deep inside a thick object to check whether the ER technique can correctly predict the ultrasonic beam decay or not.

\section{APPENDIX A COEFFICIENTS AND FUNCTIONS FOR THE CALCULATION OF AP- PROXIMATE WAVE SO- LUTION}

Coefficients and functions required to calculate the displacement field in a target material using approximate wave solution as shown in Eq. (3) are presented in this section.

Directivity function $K_{\gamma}\left(\theta_{i, 1}^{L}\right)$ for L- and T-modes:

$$
\begin{aligned}
K_{L}\left(\theta_{i, 1}^{L}\right) & =\frac{\cos \theta_{i, 1}^{L} \kappa_{1}^{2}\left(\kappa_{1}^{2} / 2-\sin ^{2}\left(\theta_{i, 1}^{L}\right)\right)}{2 G\left(\sin \theta_{i, 1}^{L}\right)}, \\
K_{T}\left(\theta_{i, 1}^{L}\right) & =\frac{\kappa_{1}^{3} \cos \theta_{i, 1}^{L} \sin \theta_{i, 1}^{L} \sqrt{1-\kappa_{1}^{2} \sin ^{2}\left(\theta_{i, 1}^{L}\right)}}{2 G\left(\kappa_{1} \sin \theta_{i, 1}^{L}\right)}
\end{aligned}
$$

where $\kappa_{1}=c_{L, 1} / c_{T, 1} . G(x)$ is the function written as follows:

$$
G(x)=\left(x^{2}-\kappa_{1}^{2} / 2\right)^{2}+x^{2} \sqrt{1-x^{2}} \sqrt{\kappa_{1}^{2}-x^{2}} .
$$

Transmission coefficient from solid 1 to 2 for the wave mode $\gamma$ in solid $2, T_{12}^{\gamma ; L}$ for L- and T-modes:

$$
\begin{aligned}
T_{12}^{L ; L} & =\frac{2 \cos \theta_{i, 1}^{L}\left(1-2 \sin ^{2} \theta_{i, 1}^{T}\right)\left(1-2 \sin ^{2} \theta_{i, 2}^{T}\right)}{\Delta_{1}+\Delta_{2}}, \\
T_{12}^{T ; L} & =\frac{-4 \sin \theta_{i, 2}^{T} \cos \theta_{i, 1}^{L} \cos \theta_{i, 2}^{L}\left(1-2 \sin ^{2} \theta_{i, 1}^{T}\right)}{\Delta_{1}+\Delta_{2}}
\end{aligned}
$$


where $\Delta_{1}$ and $\Delta_{2}$ are defined in Eqs.(A.6) and (A.7) ${ }^{29)}$ as follows:

$$
\begin{aligned}
\Delta_{1}= & \cos \theta_{i, 2}^{L}\left[1-4 \sin ^{2} \theta_{i, 1}^{T} \cos ^{2} \theta_{i, 1}^{T}+\right. \\
& \left.4 \frac{c_{T, 1}^{2}}{c_{L, 1}^{2}} \sin \theta_{i, 1}^{T} \cos \theta_{i, 1}^{T} \sin \theta_{i, 1}^{L} \cos \theta_{i, 1}^{L}\right], \\
\Delta_{2}= & \frac{\rho_{2} c_{L, 2}}{\rho_{1} c_{L, 1}} \cos \theta_{i, 1}^{L}\left[1-4 \sin ^{2} \theta_{i, 2}^{T} \cos ^{2} \theta_{i, 2}^{T}+\right. \\
& \left.4 \frac{c_{T, 2}^{2}}{c_{L, 2}^{2}} \sin \theta_{i, 2}^{T} \cos \theta_{i, 2}^{T} \sin \theta_{i, 2}^{L} \cos \theta_{i, 2}^{L}\right] .
\end{aligned}
$$

$\Delta_{x}^{\gamma ; L}$ and $\Delta_{y}^{\gamma ; L}$ in Eq. (3) are derived from a stationary phase method, as shown by Eqs. (A.8) and (A.9), respectively.

$$
\begin{gathered}
\Delta_{x}^{\gamma ; L}=r_{i, 1}^{L}+\frac{c_{\gamma, 2} \cos ^{2} \theta_{i, 1}^{\gamma}}{c_{L, 1} \cos ^{2} \theta_{i, 2}^{\gamma}} r_{i, 2}^{\gamma}, \\
\Delta_{y}^{\gamma ; L}=r_{i, 1}^{L}+\frac{c_{\gamma, 2}}{c_{L, 1}} r_{i, 2}^{\gamma}
\end{gathered}
$$

Transmission coefficient for the receiving route:

$$
T_{21}^{L ; \gamma}=\frac{\rho_{1} c_{L, 1} \cos \theta_{j, 2}^{\gamma}}{\rho_{2} c_{\gamma, 2} \cos \theta_{j, 1}^{L}} T_{12}^{\gamma ; L} .
$$

\section{APPENDIX B STRUCTURAL SIMILAR- ITY (SSIM) INDEX}

Various methods such as mean squared error and peak signal-to-noise ratio were the traditional image quality metrics until Structural SIMilarity (SSIM) Index was proposed in 2004 by Zhou Wang et al. ${ }^{31), 32)}$ SSIM is proven to be highly correlated with $\mathrm{HVS}^{31)}$ and it has gained widespread popularity as a tool to assess quality of images and also to evaluate the performance of image processing algorithms and system. ${ }^{33)}$

SSIM requires a reference image that is an undistorted image to be used as a benchmark in comparison with distorted images. Suppose $\boldsymbol{x}$ and $\boldsymbol{y}$ are two non-negative images and consider $\boldsymbol{x}$ as an image with perfect quality and $\boldsymbol{y}$ as a distorted image. The SSIM of image $\boldsymbol{y}$ compared with $\boldsymbol{x}$ can be calculated by the following equation:

$$
\operatorname{SSIM}(\boldsymbol{x}, \boldsymbol{y})=\{l(\boldsymbol{x}, \boldsymbol{y})\}^{\alpha} \cdot\{c(\boldsymbol{x}, \boldsymbol{y})\}^{\beta} \cdot\{s(\boldsymbol{x}, \boldsymbol{y})\}^{\gamma}
$$

where $\alpha, \beta$, and $\gamma$ in Eq. (B.1) are indices that can be used to bias three comparison functions. In this paper, we do not use biased comparison, so $\alpha=\beta=\gamma=1$. Comparison functions for luminance $l(\boldsymbol{x}, \boldsymbol{y})$, contrast $c(\boldsymbol{x}, \boldsymbol{y})$ and structure $s(\boldsymbol{x}, \boldsymbol{y})$ are defined as follows:

$$
\begin{aligned}
l(\boldsymbol{x}, \boldsymbol{y}) & =\frac{2 \mu_{x} \mu_{y}+C_{1}}{\mu_{x}^{2}+\mu_{y}^{2}+C_{1}}, \quad C_{1}=\left(K_{1} L\right)^{2}, \\
c(\boldsymbol{x}, \boldsymbol{y}) & =\frac{2 \sigma_{x} \sigma_{y}+C_{2}}{\sigma_{x}^{2}+\sigma_{y}^{2}+C_{2}}, \quad C_{2}=\left(K_{2} L\right)^{2}, \\
s(\boldsymbol{x}, \boldsymbol{y}) & =\frac{\sigma_{x y}+\left(C_{2} / 2\right)}{\sigma_{x} \sigma_{y}+\left(C_{2} / 2\right)} .
\end{aligned}
$$

In the abovementioned equations, $C_{1}$ and $C_{2}$ are small constants introduced to avoid instability when the denominators are very close to zero. $K_{1}$ and $K_{2}$ are arbitrary small constants, and $L$ is the dynamic range of the pixel values. Constants used in MSSIM calculation throughout this paper are $K_{1}=0.01, K_{2}=0.03$, and $L=1$. The values $\mu_{x}$ and $\mu_{y}$ in Eq. (B.2) are mean intensities of images defined as follows:

$$
\mu_{x}=\frac{1}{N} \sum_{i=1}^{N} x_{i}, \quad \mu_{y}=\frac{1}{N} \sum_{i=1}^{N} y_{i}
$$

where $N$ is the total number of pixels, and $\sigma_{x}$ and $\sigma_{y}$ are standard deviations of each image, which can be calculated from Eq. (B.6).

$$
\begin{aligned}
& \sigma_{x}=\left(\frac{1}{N-1} \sum_{i=1}^{N}\left(x_{i}-\mu_{x}\right)^{2}\right)^{1 / 2}, \\
& \sigma_{y}=\left(\frac{1}{N-1} \sum_{i=1}^{N}\left(y_{i}-\mu_{y}\right)^{2}\right)^{1 / 2} .
\end{aligned}
$$

In Eq. (B.4), $\sigma_{x y}$ is the correlation coefficient of images $\boldsymbol{x}$ and $\boldsymbol{y}$, defined as

$$
\sigma_{x y}=\frac{1}{N-1} \sum_{i=1}^{N}\left(x_{i}-\mu_{x}\right)\left(y_{i}-\mu_{y}\right) .
$$

Using all abovementioned equations, the SSIM index between two images of $\boldsymbol{y}$ and $\boldsymbol{x}$ can be computed at any pixel. If two images are perfectly the same, then $\operatorname{SSIM}(\boldsymbol{x}, \boldsymbol{y})$ is 1 . Otherwise $\operatorname{SSIM}(\boldsymbol{x}, \boldsymbol{y})<1$. For large differences between $\boldsymbol{x}$ and $\boldsymbol{y}$, the value of $[1-\operatorname{SSIM}(\boldsymbol{x}, \boldsymbol{y})]$ becomes large.

\section{REFERENCES}

1) AASHTO LRFD Bridge Design Specifications, $6^{\text {th }}$ Edition, American Association of State Highway and Transportation Officials, Washington DC, 2012.

2) ACI Committee 345: Guide for Maintenance of Concrete Bridge Members, American Concrete Institute, Michigan, 2006.

3) Zhang, W. and Yuan, H.: Corrosion fatigue effects on life estimation of deteriorated bridges under vehicle impacts, Engineering Structures, Vol. 71, pp. 128136, 2014.

4) Wenzel, H.: Health Monitoring of Bridges, John Wiley \& Sons, United Kingdom, 2009.

5) Achenbach, J. D.: Quantitative nondestructive evaluation, International Journal of Solids and Structures, Vol. 37, pp. 13-27, 2000. 
6) Berthold, B.: Color Atlas of Ultrasound Anatomy, Thieme Medical Publishers, New York, 2011.

7) Frederick, J. R., Seydel, J. A. and Fairchild, R. C.: Improved ultrasonic non-destructive testing of pressure vessels, US Nuclear Regulatory Commission Report, NUREG-0007-1, University of Michigan, Ann Arbor, Michigan, 1976.

8) Seydel, J. A.: Ultrasonic synthetic aperture focusing techniques in NDT, Research Techniques in Nondestructive Testing, Vol. 6, pp. 1-47, 1983.

9) Doctor, S. R., Hall, T. E. and Reid, L. D.: SAFTThe evolution of a signal processing technology for ultrasonic testing, NDT International, Vol. 19, No. 3, pp. 163-167, 1986.

10) Thomson, R. N.: Transverse and longitudinal resolution of the synthetic aperture focusing technique, $\mathrm{Ul}$ trasonics, Vol. 22, Issue 1, pp. 9-15, 1984.

11) Schmitz, V., Chakhlov, S. and Muller, W.: Experiences with synthetic aperture focusing technique in the field, Ultrasonics, Vol. 38, pp. 731-738, 2000.

12) Lamarre, A., Moles, M. and Lupien, V.: Phased array ultrasonic inspection of friction stir weldments, In: D. O. Thompson, D. E. Chimenti (Eds.), Review of Progress in Quantitative Nondestructive Evaluation 19, Plenum, New York, pp. 1333-1348, 1999.

13) Drinkwater, B. W. and Wilcox, P. D.: Ultrasonic arrays for non-destructive evaluation: A review, NDTEE International, Vol. 39, pp. 525-541, 2006.

14) Light, E. D., Lieu, V., Suhocki, P., Wolf, P. D. and Smith, S. W.: Progress in ring array transducers for real-time 3D ultrasound guidance of cardiac interventional devices, Ultrasonic Imaging, Vol. 33, No. 3, pp. 197-204, 2011.

15) Novotny, P. M., Stoll, J. A., Vasilyev, N. V., Del Nido, P. J., Dupont, P. E., Zickler, T. E. and Howe, R. D.: GPU-based real-time instrument tracking with threedimensional ultrasound, Medical Image Analysis, Vol. 11, pp. 458-464, 2007.

16) Linguraru, M. G., Kabla, A., Marx, G. R., Del Nido, P. J. and Howe, R. D.: Real-time tracking and shape analysis of atrial septal defects in 3D echocardiography, Academic Radiology, Vol. 14, No. 11, pp. 12981309, 2007.

17) Lingvall, F., Olofsson, T. and Stepinski, T.: Synthetic aperture imaging using sources with finite aperture: Deconvolution of the spatial impulse response, J. Acoust. Soc. Am., Vol. 114, No. 1, pp. 225-234, 2003.

18) Stepinski, T.: An implementation of synthetic aperture focusing technique in frequency domain, IEEE Transactions on Ultrasonics, Ferroelectrics, and Frequency Control, Vol. 54, No. 7, pp. 1399-1408. 2007.

19) Matsuoka, C., Nakahata, K., Baba, A., Kono, N. and Hirose, S.: Comparative study on ultrasonic imaging methods with array transducers, In: D. O. Thompson and D. E. Chimenti (Eds.), Review of Progress in Quantitative Nondestructive Evaluation 35, AIP Conference Proceedings, Vol. 975, pp. 707-714, 2008.

20) Bazulin, E. G.: Imaging of flaws with allowance for multiple reflections of ultrasonic pulses from planeparallel boundaries of a tested object, Russian Journal of Nondestructive Testing, Vol. 43, No.7, pp. 48-70, 2007.

21) Bazulin, E. G.: Obtaining flaws images that take the effect of multiple ultrasonic pulse reflections from the boundaries of a test object into account, Russian Journal of Nondestructive Testing, Vol. 46, No. 10, pp. 3458, 2010.

22) Spies, M., Rieder, H., Dillhofer, A., Schmitz, V. and Muller, W.: Synthetic aperture focusing and timeof-flight diffraction ultrasonic imaging-past and present, J. Nondestruct. Eval., Vol. 31, Issue 4, pp. 310-323, 2012.

23) Schmerr Jr, L. W.: Fundamentals of Ultrasonic Nondestructive Evaluation-A Modeling Approach, Plenum Press, New York, 1998.

24) Hirose, S., Nishimoto, S., Maruyama, T. and Nagata, Y.: Improved ultrasonic SAFT imaging of flaws in structures with curved surfaces, In: D. E. Chimenti, L. J. Bond and D. O. Thompson (Eds.), Review of Progress in Quantitative Nondestructive Evaluation 36, AIP Conference Proceedings, Vol. 1581, pp. 3641, 2014.

25) Padungsriborworn, W., Furukawa, A. and Hirose, S.: Implementation of approximate wave solution in fluid-solid two-phase media: a case study on imaging of aluminum rod with side-drilled holes, Journal of Nondestructive Evaluation, Vol. 34, 2015.

26) Digital Imaging Tutorial, http://www.library. cornell.edu/preservation/tutorial/intro/ intro-01.html, Cornell University, accessed 25 December 2014.

27) Hutchins, D. A. and Hayward, G.: Radiated fields of ultrasonic transducers, Physical Acoustics: Ultrasonic Measurement Methods, Vol. 19, pp. 1-80, 1990.

28) Ding, D., Zhang, Y. and Liu, J.: Some extensions of the Gaussian beam expansion: Radiation fields of the rectangular and the elliptical transducer, J. Acoust. Soc. Am., Vol. 113, No.6, pp. 3043-3048, 2003.

29) Huang, R., Schmerr Jr, L. W. and Sedov, A.: Modeling the radiation of ultrasonic phased-array transducers with gaussian beams, IEEE Transactions on Ultrasonics, Ferroelectrics, and Frequency Control, Vol. 55, No. 12, pp. 2692-2702, 2008.

30) Schmerr Jr, L. W. and Song, J. S.: Ultrasonic Nondestructive Evaluation Systems: Models and Measurements, Springer, New York, 2007.

31) Wang, Z., Bovik, A. C., Sheikh, H. R. and Simoncelli, E. P.: Image quality assessment: From error visibility to structural similarity, IEEE Transactions on Image Processing, Vol. 13, No.4, pp. 600-612, 2004.

32) The SSIM Index for Image Quality Assessment, https://ece.uwaterloo.ca/ z70wang/ research/ssim/, University of Waterloo, accessed 25 December 2014.

33) Brunet, D., Vrscay, E. R. and Wang, Z.: On the mathematical properties of the structural similarity index, IEEE Transactions on Image Processing, Vol. 21, No.4, pp. 1488-1499, 2012.

(Received March 11, 2015) 